Louisiana State University

LSU Digital Commons

$1-1-1998$

\title{
Macroscopic studies of short-pulse high-order harmonic generation using the time-dependent Schrödinger equation
}

\author{
M. B. Gaarde \\ Lunds Tekniska Högskola \\ Ph Antoine \\ Université Catholique de Louvain
}

A. L'Huillier

Lunds Tekniska Högskola

K. J. Schafer

Louisiana State University

K. C. Kulander

Lawrence Livermore National Laboratory

Follow this and additional works at: https://digitalcommons.Isu.edu/physics_astronomy_pubs

\section{Recommended Citation}

Gaarde, M., Antoine, P., L'Huillier, A., Schafer, K., \& Kulander, K. (1998). Macroscopic studies of short-pulse high-order harmonic generation using the time-dependent Schrödinger equation. Physical Review A Atomic, Molecular, and Optical Physics, 57 (6), 4553-4560. https://doi.org/10.1103/PhysRevA.57.4553

This Article is brought to you for free and open access by the Department of Physics \& Astronomy at LSU Digital Commons. It has been accepted for inclusion in Faculty Publications by an authorized administrator of LSU Digital Commons. For more information, please contact ir@lsu.edu. 


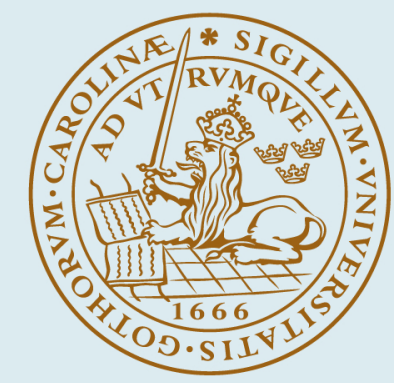

\title{
LUND UNIVERSITY
}

\section{Macroscopic studies of short-pulse high-order harmonic generation using the time- dependent Schrodinger equation}

\author{
Gaarde, Mette; Antoine, P; L'Huillier, Anne; Schafer, K. J; Kulander, K. C \\ Published in: \\ Physical Review A (Atomic, Molecular and Optical Physics)
}

DOI:

10.1103/PhysRevA.57.4553

1998

Link to publication

Citation for published version (APA):

Gaarde, M., Antoine, P., L'Huillier, A., Schafer, K. J., \& Kulander, K. C. (1998). Macroscopic studies of shortpulse high-order harmonic generation using the time-dependent Schrodinger equation. Physical Review $A$ (Atomic, Molecular and Optical Physics), 57(6), 4553-4560. https://doi.org/10.1103/PhysRevA.57.4553

Total number of authors:

5

\footnotetext{
General rights

Unless other specific re-use rights are stated the following general rights apply:

Copyright and moral rights for the publications made accessible in the public portal are retained by the authors and/or other copyright owners and it is a condition of accessing publications that users recognise and abide by the legal requirements associated with these rights.

- Users may download and print one copy of any publication from the public portal for the purpose of private study or research.

- You may not further distribute the material or use it for any profit-making activity or commercial gain

- You may freely distribute the URL identifying the publication in the public portal

Read more about Creative commons licenses: https://creativecommons.org/licenses/

Take down policy

If you believe that this document breaches copyright please contact us providing details, and we will remove access to the work immediately and investigate your claim.
} 


\title{
Macroscopic studies of short-pulse high-order harmonic generation using the time-dependent Schrödinger equation
}

\author{
M. B. Gaarde, ${ }^{1,2} \mathrm{Ph}$. Antoine, ${ }^{3}$ A. L'Huillier, ${ }^{1}$ K. J. Schafer, ${ }^{4}$ and K. C. Kulander ${ }^{5}$ \\ ${ }^{1}$ Department of Physics, Lund Institute of Technology, P.O. Box 118, S-22100 Lund, Sweden \\ ${ }^{2}$ Niels Bohr Institute, Orsted Laboratory, Universitetsparken 5, 2100 Kbh Ø, Denmark \\ ${ }^{3}$ Laboratoire de Physique Atomique et Moléculaire, Université Catholique de Louvain, 2 Chemin du Cyclotron, \\ B-1348 Louvain-la-Neuve, Belgium \\ ${ }^{4}$ Department of Physics and Astronomy, Louisiana State University, Baton Rouge, Louisiana 70803-4001 \\ ${ }^{5}$ TAMP Group, Lawrence Livermore National Laboratory, Livermore, California 94551
}

(Received 3 December 1997)

\begin{abstract}
We consider high harmonic generation by ultrashort (27-108 fs) laser pulses and calculate the macroscopic response of a collection of atoms to such a short pulse. We show how the harmonic spectrum after propagation through the medium is significantly different from the single-atom spectrum. We use single-atom data calculated by integration of the time-dependent Schrödinger equation and propose a method, based on an adiabatic approximation, to extract the data necessary to perform a propagation calculation. [S1050-2947(98)00306-0]
\end{abstract}

PACS number(s): $32.80 . \mathrm{Rm}, 42.65 . \mathrm{Ky}$

\section{INTRODUCTION}

The recent technological progress of short-pulse highpower lasers based on titanium-sapphire crystals has made it possible to generate high-order harmonics with laser pulses of only a few optical cycles (with duration below $30 \mathrm{fs}$ ) [1-3]. The harmonic spectra present a characteristic behavior: a plateau region where all the harmonics have nearly the same strength and a cutoff region where the harmonic strength decreases rapidly with the order. Harmonic spectra generated with these short laser pulses extend very far into the soft-x-ray range, with maximum energies exceeding 300 $\mathrm{eV}$. Using laser pulses $27 \mathrm{fs}$ in duration, Zhou et al. [1] have generated well-resolved harmonics up to the 211th order of $800-\mathrm{nm}$ radiation $(310 \mathrm{eV})$, i.e., into the "water window." Spielmann et al. [3], using 5-fs laser pulses, have also observed harmonic radiation in this spectral region. The spectrum, however, does not consist of resolved peaks at the harmonic frequencies, but of a broad continuum from the 13th harmonic.

These recent results raise several questions. What is the spectral width of the harmonics? From which pulse duration and from which order do consecutive harmonics begin to overlap? Calculations of the emission from a single atom exposed to a short, intense, laser pulse show rather noisy and almost continuous spectra in the plateau region, the harmonic structure reappearing in the cutoff [4-6]. This is true even for relatively long pulses, of duration $100 \mathrm{fs}$, which is at variance with experimental results. A complete theoretical description of harmonic-generation processes requires of course one to account for propagation in the nonlinear medium. Can propagation effects "clean up" single-atom harmonic spectra in the plateau region so that, at least under some conditions, it is possible to obtain well-resolved harmonic peaks?

Another question is the role of dynamical effects in the generation of harmonics by these extremely short laser pulses. With the very short laser pulses now available, the intensity of the driving field changes significantly during each optical cycle.
The understanding of the high-harmonic-generation process has been progressing considerably during the past five years, largely owing to the development of the semiclassical model $[7,8]$. In this description one considers the atom to have only one electron and only one bound state: the ground state. In the presence of the strong field, the Coulomb potential can be "bent" and the electron can be released from the nucleus by tunneling ionization. Once in the continuum it is accelerated by the field, and when the field changes sign, the electron can be pushed back towards the nucleus and may recombine, thereby releasing a photon. Fully quantummechanical formulations of this model, known as the strongfield approximation (SFA), have been given $[9,10]$. For a harmonic belonging to the plateau region, there are several electronic trajectories that contribute to the generation process, giving rise to different, interfering, contributions to the dipole moment of some particular harmonic [11]. The phase of the emitted radiation is locked to the electronic motion in the continuum and the different trajectories therefore contribute with different phase behavior. One of these trajectories contributes with a phase that varies quite slowly as a function of the laser intensity, whereas the others in general exhibit a rapidly decreasing phase. When calculating the macroscopic response of the atoms in a gas jet, by propagating the generated radiation through the medium, phase matching becomes essential. Since the different trajectories have different phase responses they will not be phase matched simultaneously $[12,13]$. Depending on the focusing conditions, one phase behavior or another can be "selected" by phase matching.

A propagation calculation requires the knowledge of the single-atom dipole strength (and phase) as a function of the intensity of the driving field, for a large number of intensity points (typically a few hundreds), in order to be able to account for the intensity distribution in the laser focus. This explains why most of the calculations have been performed using simple models for describing harmonic generation, such as the SFA. However, the SFA is mainly valid for highorder harmonics and often underestimates the harmonic strengths, compared to "exact" results, obtained by numeri- 
cal integration of the time-dependent Schrödinger equation (TDSE).

In the present work we propose a method for performing propagation calculations using single-atom data obtained by integrating the TDSE for a laser pulse. The single-atom harmonic spectrum is the result of what has happened during the entire pulse and therefore contains information about the process for several intensities, those spanned during the laser pulse. By Fourier transforming the harmonic field (as a function of frequency) into the time domain and by relating time to the instantaneous laser intensity, it is possible to obtain the harmonic strength (amplitude and phase) as a function of the laser intensity, which is then used in a propagation calculation. We here assume that the main features of the generation process are determined by the instantaneous intensity of the field and not so much by the dynamics. In other words, we are making an adiabatic approximation. We investigate how propagation changes the aspect (in particular the ratio between harmonic peaks and background) and length of the plateau in the harmonic spectrum.

This paper is organized as follows. In Sec. II we describe our theoretical method. We use single-atom spectra obtained in argon at different laser intensities and for two pulse durations: $108 \mathrm{fs}$ and $27 \mathrm{fs}$. The laser wavelength is $810 \mathrm{~nm}$. In Sec. III we present numerical results for two harmonics: the 49th harmonic, in the cutoff region, and the 27th harmonic, in the plateau region. Finally we discuss our results in Sec. IV.

\section{THEORETICAL APPROACH}

We consider the interaction of an atom with an intense linearly polarized laser field (in the $x$ direction) and integrate the TDSE within the framework of the single-active-electron approximation. (For details, see [14]). The laser field is pulsed with a $\cos ^{2}$ envelope and a pulse length of $\tau$. The emitted radiation spectrum is proportional to the Fourier transform of the acceleration $a(t)$ of the active electron, given by

$$
a(t)=\frac{d^{2}\langle x\rangle}{d t^{2}}=-\langle\psi|[H,[H, x]]| \psi\rangle
$$

The dipole spectrum is obtained by Fourier transforming the time-dependent acceleration as

$$
D(\omega)=\frac{1}{\tau} \frac{1}{\omega^{2}} \int_{-\infty}^{\infty} a(t) \exp (i \omega t) d t
$$

Calculations have been performed in argon using a wavelength $\lambda=810 \mathrm{~nm}$, two pulse durations $\tau=108$ and $27 \mathrm{fs}$, and several laser peak intensities, 1.5, 2, 2.5, 3, and $3.5 \times 10^{14} \mathrm{~W} / \mathrm{cm}^{2}$. As shown below, the use of data obtained at different peak intensities allows us to test the validity of the numerical method and hence of the adiabatic approximation.

In Fig. 1 we show spectra resulting from two calculations in argon with peak intensity $3 \times 10^{14} \mathrm{~W} / \mathrm{cm}^{2}$ and pulse lengths $\tau=108$ fs [Fig. 1(a)] and 27 fs [Fig. 1(b)], respectively. Shown on the abscissa is the photon energy in units of the laser frequency $\omega_{0}$. In both spectra, but especially in the

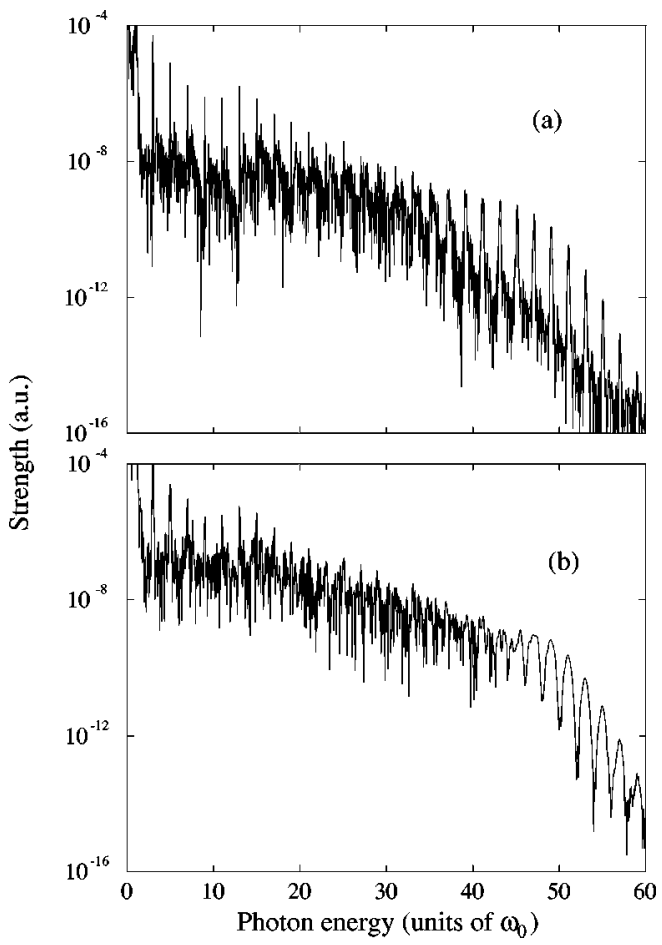

FIG. 1. Emission spectra from an argon atom submitted to a laser field with wavelength $\lambda=810 \mathrm{~nm}$, peak intensity $3 \times 10^{14}$ $\mathrm{W} / \mathrm{cm}^{2}$, and pulse length (a) $\tau=108 \mathrm{fs}$ and (b) $\tau=27 \mathrm{fs}$.

one obtained at the shortest pulse duration, there is hardly any harmonic structure left in the plateau, due to a very high background. In contrast, the harmonics are well defined in the cutoff. These harmonics have a regular, almost quadratic, phase behavior. This led to the idea of recompressing the harmonic pulse to a duration of less than 2 fs by getting rid of the linear chirp, which was presented in $[4,15]$.

We now focus our attention on a particular harmonic, with frequency $q \omega_{0}$. We calculate its time profile by inverse Fourier transforming its spectrum, multiplied by a window function $F$ centered around the harmonic frequency:

$$
D_{q}(t)=\frac{1}{N} \int_{-\infty}^{\infty} D(\omega) \exp (-i \omega t) F\left(\omega-q \omega_{0}\right) d \omega
$$

$N$ is a normalizing factor, which is proportional to the full width at half maximum (FWHM) of the harmonic. We use two different window functions in the following. One is a broad function with width $2 \omega_{0}$, whose aim is simply to separate the different harmonic components. We have chosen this window to be of square shape and have checked that the sharp edges of the window function do not influence significantly the results obtained. The other function is much narrower and is used to pick up only the harmonic component of the generated radiation and remove the background. In this case we use a Gaussian window function of width $0.75 \omega_{0}$. At every point in time $t$, we know the intensity of the driving field:

$$
I(t)=I_{0} \cos ^{2}\left(\frac{\pi}{2} \frac{t}{\tau}\right)
$$


$I_{0}$ denoting the peak intensity. From $D_{q}(t)$ we obtain then easily $D_{q}(I)$. By doing the normalization in Eq. (3) we can compare directly $\left|D\left(q \omega_{0}\right)\right|^{2}$ obtained with a laser pulse having a given peak intensity $I_{0}$ with $\left|D_{q}\left(I=I_{0}\right)\right|^{2}$, coming from single-atom data corresponding to a different (higher) peak intensity. We use only the first half of the pulse since, due to the influence of ionization, the time profile of the harmonic is not symmetric, the main part of the radiation being generated on the rising edge of the pulse.

Having obtained the dipole strength as a function of the laser intensity, we proceed to propagate the individual harmonics through the density distribution within the gas jet. Our approach to propagation has been presented in detail in [16] and we shall only describe it briefly here. We integrate the propagation equation for each harmonic component in the paraxial and slowly varying envelope approximation. We include ionization in the single-atom response, which means that depletion is accounted for in an approximate way (since we consider only the rising edge of the pulse), but we neglect dispersion effects, in particular those induced by ionization. This means that our calculations are strictly valid in the limit of low atomic density. In this approximation, the fundamental field is not modified as it propagates through the medium and the propagation equation for the $q$ th harmonic field reads

$$
\nabla_{\perp}^{2} E_{q}(\vec{r}, t)+\frac{2 i q \omega_{0}}{c} \frac{\partial E_{q}(\vec{r}, t)}{\partial z}=-\frac{q^{2} \omega_{0}^{2}}{\epsilon_{0} c^{2}} P_{q}^{N L}(\vec{r}, t),
$$

where $z$ denotes the direction of propagation, $E_{q}(\vec{r}, t)$ is the (complex) slowly varying envelope of the harmonic field, and $P_{q}^{N L}(\vec{r}, t)$ the laser-induced nonlinear polarization equal to $2 \mathcal{N}_{a}(z) D_{q}\left[I_{1}(\vec{r}, t)\right] \exp \left[i q \phi_{1}(\vec{r}, t)\right] . \mathcal{N}_{a}(z)$ denotes the initial atomic density. $I_{1}(\vec{r}, t)$ and $\phi_{1}(\vec{r}, t)$ are the intensity and phase of the fundamental laser field envelope. The laser beam is assumed to be Gaussian, with a Gaussian temporal envelope. The time $t$ refers to the (slow) variation of the pulse envelope. The integrated power spectrum for each harmonic is obtained by Fourier transforming the timedependent field and integrating over the transverse direction $r$ as (we here use cylindrical coordinates)

$$
I_{q}(\omega)=\int\left|\int_{-\infty}^{\infty} E_{q}(r, z, t) \exp \left[i\left(\omega-q \omega_{0}\right) t\right] d t\right|^{2} 2 \pi r d r .
$$

$z$ is here simply chosen at the exit of the nonlinear medium. The calculations presented below have all been performed by using a fundamental Gaussian beam with a confocal parameter of $5 \mathrm{~mm}$ and for a Lorentzian gas jet with a FWHM of 1 $\mathrm{mm}$. The density in the gas jet is approximately $5 \times 10^{17}$ atoms $/ \mathrm{cm}^{3}$. In most of the calculations, we use the singleatom data obtained with the longest pulse duration, $108 \mathrm{fs}$, for which the adiabatic approximation should be the most valid, and we assume the pulse width of the (Gaussian) laser pulse that we use in the propagation calculation to be of the same width at half maximum.

\section{NUMERICAL RESULTS}

We first test our method on the 49th harmonic, which, in the single-atom spectrum, is at the beginning of the cutoff

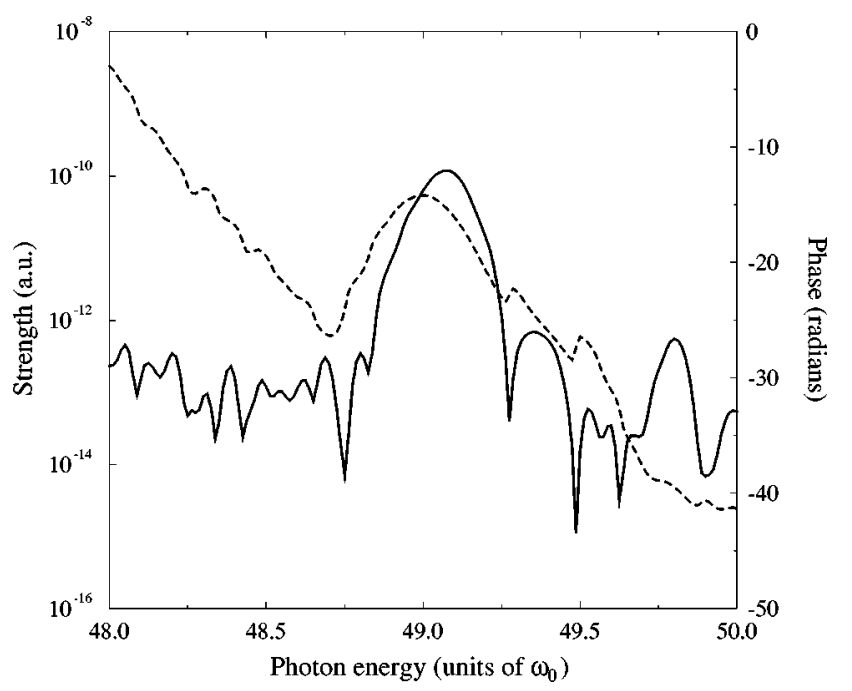

FIG. 2. Single-atom spectrum (solid line) and phase (dashed line) of the 49th harmonic, for a peak intensity of the driving field of $3 \times 10^{14} \mathrm{~W} / \mathrm{cm}^{2}$.

region at the intensity $3 \times 10^{14} \mathrm{~W} / \mathrm{cm}^{2}$. Figure 2 shows its strength (solid line) and phase (dashed line) as functions of frequency at the same laser peak intensity [the spectrum in Fig. 2 is part of the spectrum in Fig. 1(a)]. The harmonic spectrum is two orders of magnitude above the background, which allows us to use the narrow window without ambiguity. It is slightly blueshifted, an effect that we attribute to ionization: As discussed in several works $[15,17]$, the harmonic spectrum is negatively chirped, with the red part of the spectrum mostly in the falling edge of the pulse and the blue part in the leading edge. Since ionization reduces the contribution of the falling edge, the red part is depleted, which leads to a blueshift of the generated radiation. The phase variation is approximately quadratic, superposed on a rapidly decreasing background. The time profile of the 49th harmonic emitted by a single atom [see Eq.(3)] is shown in Fig. 3 for two different peak intensities: $I=3 \times 10^{14}$ and $3.5 \times 10^{14} \mathrm{~W} / \mathrm{cm}^{2}$. Ionization leads to asymmetric time profiles, especially for the highest intensity.

The corresponding single-atom intensity dependences, ob-

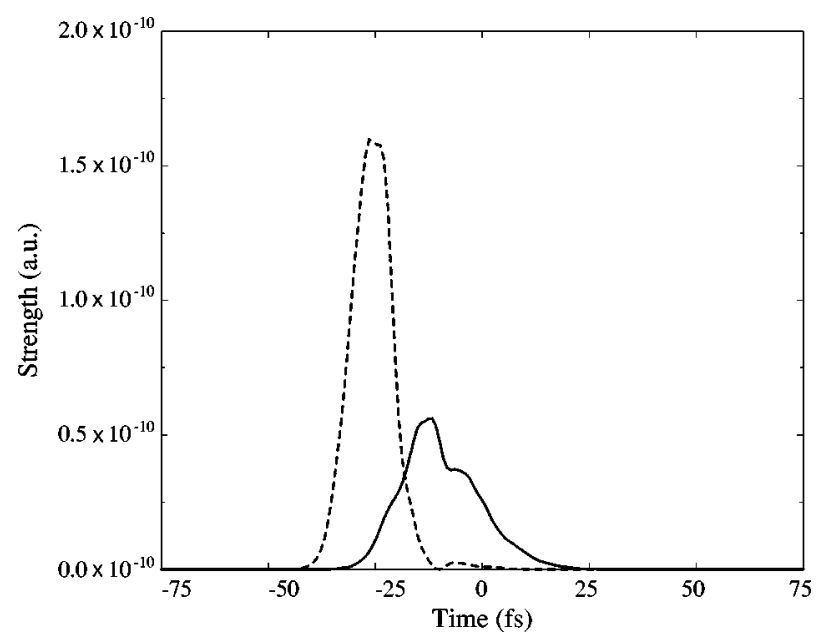

FIG. 3. Time profiles of the 49th harmonic at $I=3 \times 10^{14}$ (solid line) and $3.5 \times 10^{14}$ (dashed line) $\mathrm{W} / \mathrm{cm}^{2}$. 


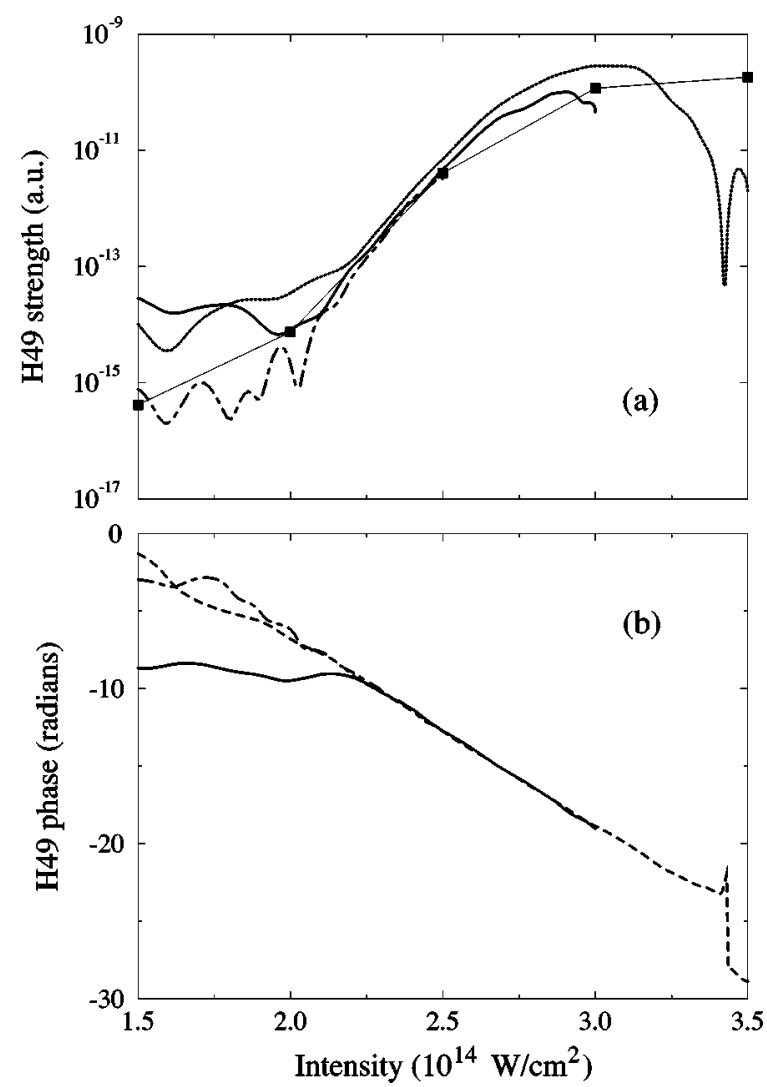

FIG. 4. Intensity variation of the (a) 49th harmonic strength and (b) phase, obtained from spectra generated at different peak intensities: $2.5 \times 10^{14}$ (dot-dashed line), $3 \times 10^{14}$ (solid line), and $3.5 \times 10^{14} \mathrm{~W} / \mathrm{cm}^{2}$ (dashed line). The dipole strengths read directly from the spectra are shown as solid squares.

tained using Eqs. (3) and (4), are shown in Figs. 4(a) and 4(b) for the dipole strength and phase, respectively. We compare data extracted from single-atom spectra obtained with three different peak intensities: $I_{0}=2.5 \times 10^{14}, 3 \times 10^{14}$, and $3.5 \times 10^{14} \mathrm{~W} / \mathrm{cm}^{2}$. Also included (with squares) are the dipole strength of the 49th harmonic read directly from these spectra, as well as from the spectra with peak intensities $I_{0}$ $=1.5 \times 10^{14}$ and $2 \times 10^{14} \mathrm{~W} / \mathrm{cm}^{2}$. The comparison between the results coming from different intensities, as well as to the directly calculated values, is a test of the validity of our method and of the adiabatic approximation. As the figure shows, this comparison is rather good for intensities between $2 \times 10^{14}$ and $3 \times 10^{14} \mathrm{~W} / \mathrm{cm}^{2}$. The discrepancy at high intensity is due to the influence of ionization and depletion of the ground state. The strength flattens out at low intensities due to the fact that the 49th harmonic becomes buried in the background for low intensities. In the propagation calculations, we only use the single-atom data for intensities above $2 \times 10^{14} \mathrm{~W} / \mathrm{cm}^{2}$ and extend the data below that intensity, using a power law given by perturbation theory.

The results obtained concerning the intensity dependence of the dipole phase agree well for the different intensities of the single-atom spectra, again above $2 \times 10^{14} \mathrm{~W} / \mathrm{cm}^{2}$. The slope of the dipole phase in this region is equal to $-2.1 U_{p} / \omega_{0}$, in excellent agreement with the value predicted by the SFA for the phase in the cutoff region [11]. We have also investigated the effect of the choice of window function and compared results obtained with the narrow and

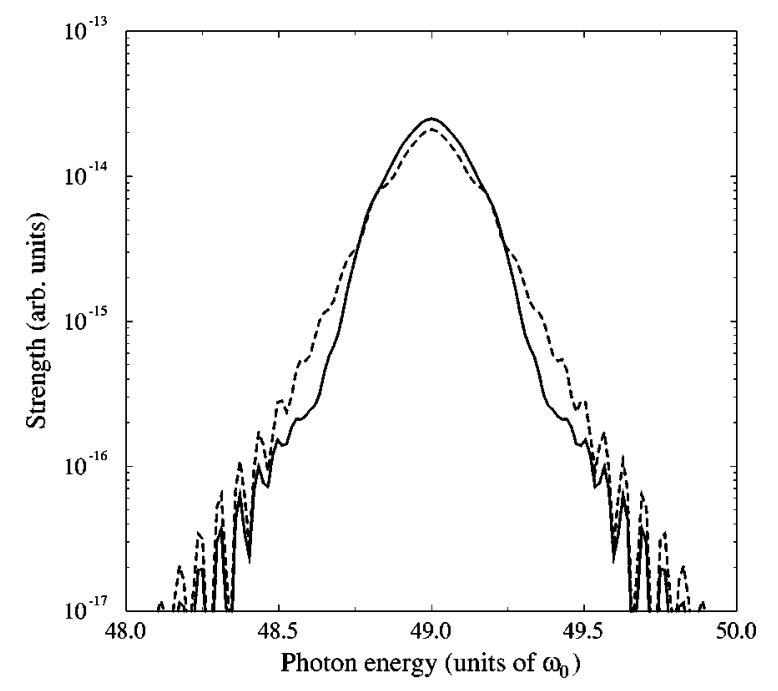

FIG. 5. Propagated spectrum for the 49th harmonic, for a peak intensity of $2.7 \times 10^{14} \mathrm{~W} / \mathrm{cm}^{2}$. The solid (dashed) line corresponds to single-atom data obtained at an intensity $I_{0}=3(3.5) \times 10^{14}$ $\mathrm{W} / \mathrm{cm}^{2}$.

the broad window. The difference in both the dipole strength and phase is very small.

In Fig. 5 we show the propagated spectrum of the 49th harmonic, calculated for a peak intensity of $2.7 \times 10^{14}$ $\mathrm{W} / \mathrm{cm}^{2}$. The focus of the laser is chosen to be in the center of the gas jet. We compare again spectra coming from two different sets of data, with peak intensities $3 \times 10^{14}$ and 3.5 $\times 10^{14} \mathrm{~W} / \mathrm{cm}^{2}$. The difference between the two spectra is quite small (note the logarithmic scale). The harmonic spectra exhibit an extremely high signal-to-background ratio (more than three orders of magnitude), higher than in the single-atom response. Indeed, the background is not as well phase matched as the harmonic components and therefore does not survive propagation. As shown below, this effect is even more pronounced for the harmonics in the plateau. The FWHM of the propagated spectrum is about $0.25 \omega_{0}$, giving a $\Delta \omega / 49 \omega_{0}$ of approximately $5 \times 10^{-3}$, which is about twice as much as in the single-atom spectrum. The additional broadening compared to the single-atom data is due to the propagation of the chirped harmonic pulse through the nonlinear medium. This aspect is discussed in detail in [15].

We have performed a similar analysis for the 27th harmonic, which is in the plateau region. In the single-atom spectrum, as shown in Fig. 6, the harmonic structure has almost completely disappeared in the background. The dashed line shows the product of the spectrum and the narrow window function used in the Fourier transform. In contrast to the case of the 49th harmonic, it is not clear whether it is correct to introduce this window function since the background in this case might be interpreted as part of the (frequency-shifted) harmonic radiation. In Fig. 7 we show intensity dependences obtained from spectra generated at different peak intensities, all obtained with the narrow window function. As for the 49th harmonic, the agreement between the different results is very good and compare well with the points read directly from the spectra (squares). Note also that the spurious flattening of the strength at low intensities, as observed previously on the 49th harmonic, happens at a lower intensity, below $10^{14} \mathrm{~W} / \mathrm{cm}^{2}$. Furthermore, we show 


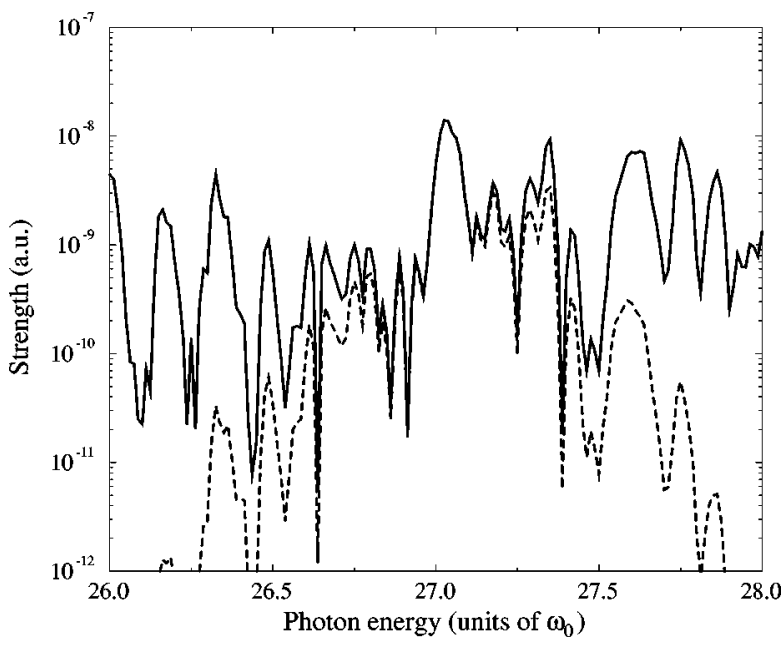

FIG. 6. Single-atom spectrum (solid line) of the 27th harmonic, for a peak intensity of the driving field of $3 \times 10^{14} \mathrm{~W} / \mathrm{cm}^{2}$. The dashed line shows the product of the spectrum and the narrow window function.

results calculated with the SFA, also in very good agreement with the TDSE data. Note, however, that this agreement is slightly artificial, since ionization is not included in the SFA calculation [18].

In Fig. 8 we show the influence of the choice of window function. The effect on the dipole strength, as shown in Fig. $8(\mathrm{a})$, of using the narrow window instead of the broad window is to somewhat smooth the curve. The phase, however, undergoes a dramatic change [Fig. 8(b)]. The phase corresponding to the narrow window is rather smooth and changes quite slowly, whereas the phase corresponding to the broad window changes much faster and with many different slopes. We stress that the "noise" on the strength, and the rapidly changing phase, actually comes from the background (or frequency shifted) radiation and is not an effect of the sharp edges of the broad square window function. We have

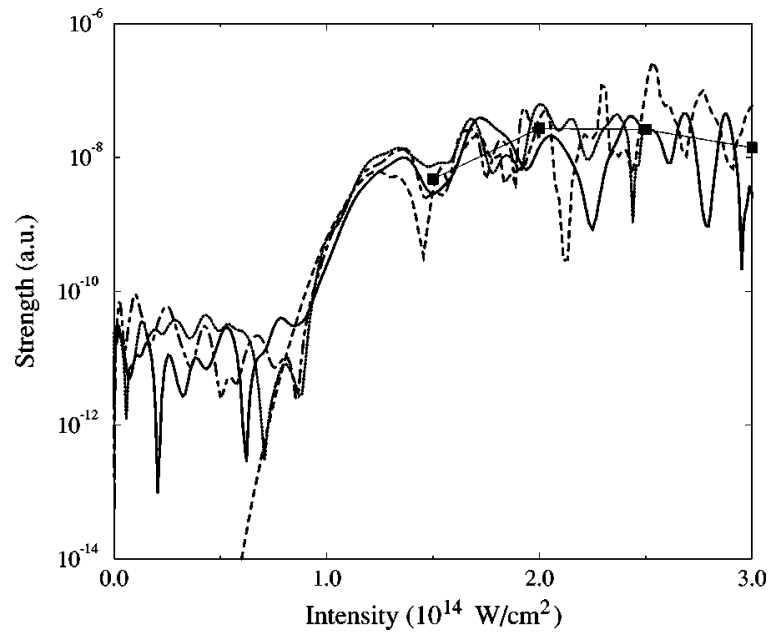

FIG. 7. Intensity variation of the 27th harmonic strength obtained from spectra generated at different peak intensities: $2 \times 10^{14}$ (dot-dashed line), $2.5 \times 10^{14}$ (dotted line), and $3 \times 10^{14}$ $\mathrm{W} / \mathrm{cm}^{2}$ (solid line). The dipole strengths read directly from the spectra are shown as solid squares. The result of the SFA is shown as a dashed line.
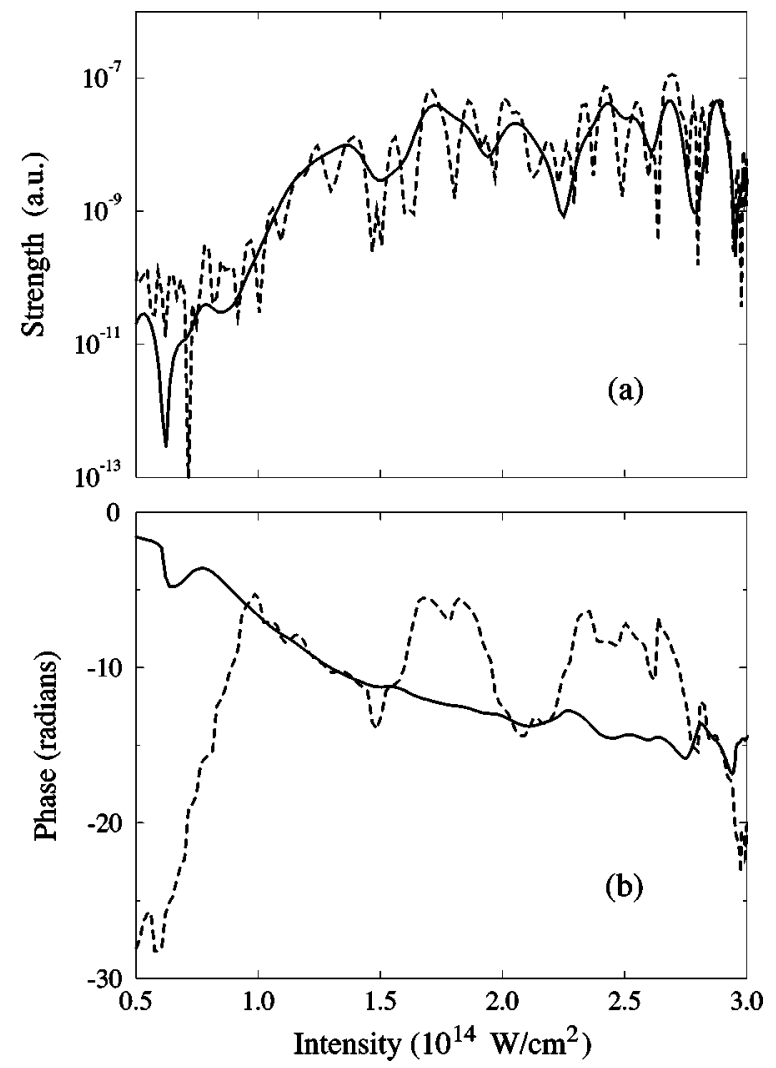

FIG. 8. Intensity variation of the (a) 27 th harmonic strength and (b) phase obtained at $3 \times 10^{14} \mathrm{~W} / \mathrm{cm}^{2}$ with a narrow window (solid line) and a broad window, of width $2 \omega_{0}$ (dashed line).

checked this by testing also a broad Gaussian window (with a FWHM of $2 \omega_{0}$ ). This gives results very similar to the ones obtained with the broad square window.

The origin of the multiple and rapid phase variations is that several electronic trajectories are contributing to the generation process, as discussed in the Introduction. These trajectories interfere and, depending on the laser intensity, one of them can become dominant and impose the phase variation [11]. The radiation close to the harmonic frequency, as selected by the narrow window, mainly results from the trajectory contributing with the relatively slow phase variation, whereas the frequency shifted radiation far away from the harmonic peak results from a much more complex electron dynamics (leading to a complicated phase variation). ${ }^{1}$

Figure 9(a) shows the propagated spectrum of the 27th harmonic at a peak intensity in the focus of $3.5 \times 10^{14}$ $\mathrm{W} / \mathrm{cm}^{2}$, where the gas jet is placed $2 \mathrm{~mm}$ after the laser focus. In the following we define $z_{0}$ as the position of the center of the gas jet relative to the laser focus; thus in this case $z_{0}=2$. The spectrum shown by the solid line comes from the data generated with the narrow window function and by the dashed line to the broad window function. Note how remarkably nice the propagated spectrum of the 27 th harmonic is. In the single-atom spectrum it is hardly distin-

\footnotetext{
${ }^{1}$ Note that, in general, we find phase variations to be more dominated by the slow phase variation than predicted from the SFA. A detailed comparison of these two approaches will be presented elsewhere.
} 


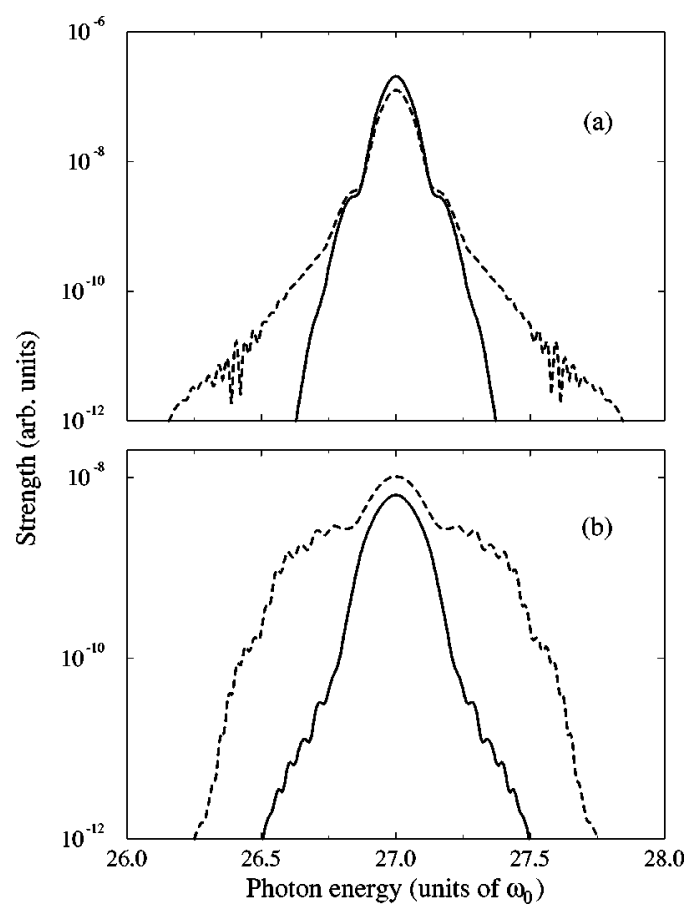

FIG. 9. Comparison of spectra from data obtained with the narrow window (solid line) and the broad window (dashed line). In (a) the gas jet is placed $2 \mathrm{~mm}$ after the focus and in (b) it is centered in the laser focus.

guishable from the background, but the harmonic profile is well defined in the propagated spectrum with a signal-tonoise ratio of more than four orders of magnitude.

The very good agreement between the two spectra shown in Fig. 9(a), in spite of their different single-atom phase behavior, can be explained as a selection by phase matching of one trajectory out of several $[12,13]$. When the gas jet is after the focus, phase matching selects slow phase variations. The single-atom data obtained with the narrow window function exhibit such a slow (as well as smooth) phase variation, which leads to good phase matching. The single-atom data obtained with the broad window show several phase behaviors. One is the same as before [see Fig. 8(b), e.g., between $1 \times 10^{14}$ and $1.5 \times 10^{14} \mathrm{~W} / \mathrm{cm}^{2}$ ) and gets well phase matched. The others are more rapid and do not survive propagation. The fact that the two spectra turn out so similar (with the same signal-to-background ratio) probably means that the slow phase variation can be associated with the nominal harmonic frequency, whereas the faster phase variationsleading to larger spectral spreading — can be associated with the frequency shifted radiation.

However, when the gas jet is centered in the focus of the laser, phase-matching conditions are different and favor trajectories with faster phase variations [12]. This is shown in Fig. 9(b), where the intensity in the center of the gas jet (and thereby in the laser focus) is chosen to be the same as in Fig. 9 (a), namely, $2.1 \times 10^{14} \mathrm{~W} / \mathrm{cm}^{2}$. In general, phase-matching conditions are worse when the focus is put in the center of the gas jet $\left(z_{0}=0\right)$ [15] and the efficiency therefore goes down compared to $z_{0}=2$. In addition, since there is no rapidly varying phase component in the data coming from the narrow window function, the efficiency goes down more in this case. This also means that the difference after propaga-

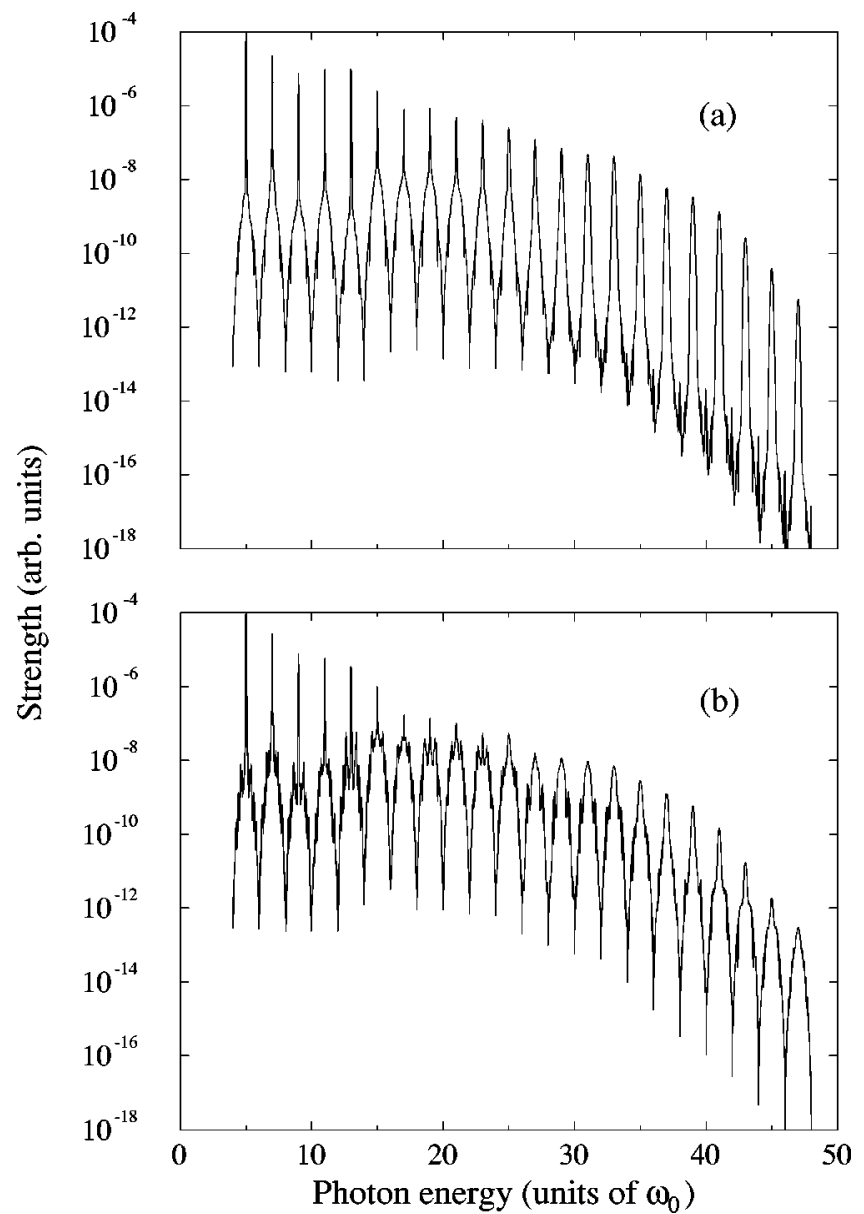

FIG. 10. Spectrum obtained with (a) $I=3.5 \times 10^{14}$ and $z_{0}=2$ and (b) $I=3 \times 10^{14} \mathrm{~W} / \mathrm{cm}^{2}$ and $z_{0}=0$.

tion between the two sets of input data is much larger, the spectrum using the broad window function having pedestals only one order of magnitude below the harmonic strength, since in this case also some of the frequency shifted radiation is phase matched. Note, however, that the wings of the spectrum do decrease rapidly to about four orders of magnitude below the peak of the harmonic.

Encouraged by the results on the 27th harmonic, we have calculated the intensity profiles for all the harmonics in the spectrum in order to propagate the entire spectrum. In Fig. 10 we show the entire spectrum after propagation. We have used a set of data having a single-atom peak intensity $3 \times 10^{14} \mathrm{~W} / \mathrm{cm}^{2}$ and applied the broad window function, in the macroscopic conditions $z_{0}=2$ and $I=3.5 \times 10^{14} \mathrm{~W} / \mathrm{cm}^{2}$ [Fig. 10(a)] and $z_{0}=0$ and $I=3 \times 10^{14} \mathrm{~W} / \mathrm{cm}^{2}$ [Fig. 10(b)]. The spectrum is remarkably nice with a signal-to-noise ratio of more than four orders of magnitude even in the plateau; the explanation for this is as presented above for the $27 \mathrm{th}$ harmonic [19].

In Fig. 11 we show the total number of photons generated in the two different focusing conditions shown in Figs. 10(a) and 10(b). The number of photons obtained, in this case $10^{5}-10^{6}$ photons in the plateau, is an important parameter for comparisons with experiments. The numbers shown in the figure are absolute values since they have been obtained using exact data for the dipole strengths. The cutoff energy of the propagated spectra corresponds approximately to $I_{p}$ 


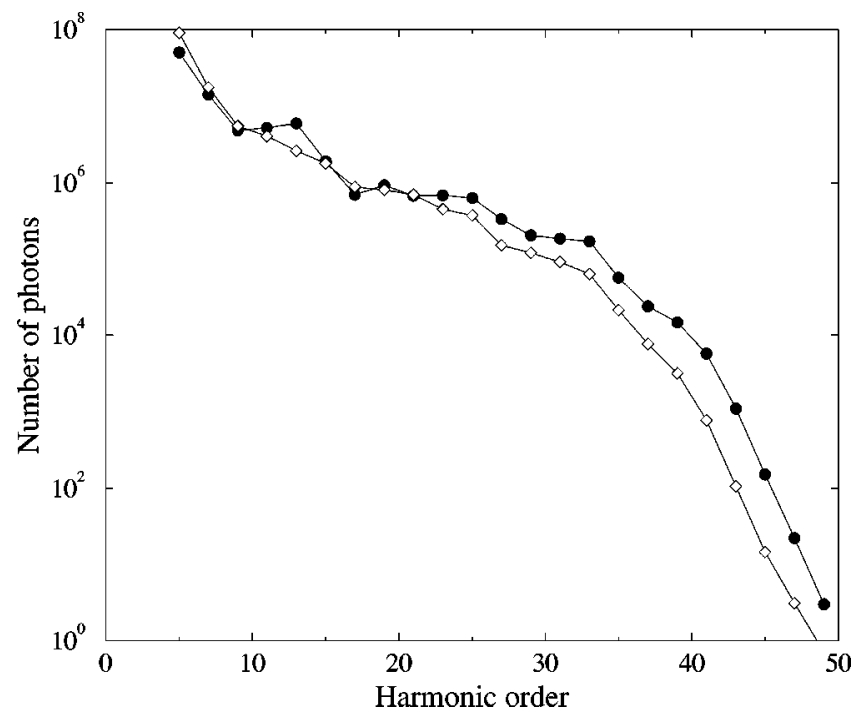

FIG. 11. Total number of photons generated in two different focusing conditions: $z_{0}=2$ and $I_{0}=3.5 \times 10^{14} \mathrm{~W} / \mathrm{cm}^{2}$ (filled symbols) and $z_{0}=0$ and $I_{0}=3 \times 10^{14} \mathrm{~W} / \mathrm{cm}^{2}$ (open symbols).

$+2 U_{p}$, which is somewhat lower than for the single atom spectra [20].

Note the difference between the results obtained in the different focusing conditions. The number of photons is generally lower when $z_{0}=0$, the difference increasing with the harmonic order, even though the intensity in the focus is actually higher in this case (since for $z_{0}=2$ the intensity in the center of the jet is $2.1 \times 10^{14} \mathrm{~W} / \mathrm{cm}^{2}$ ). This means that the better phase-matching conditions for $z_{0}=2$ play a large role for the output of the medium.

Finally, in Fig. 12 we show propagated spectra for a driving field with a pulse length of $\tau=27 \mathrm{fs}$. The data come from a single-atom spectrum with peak intensity $3 \times 10^{14} \mathrm{~W} / \mathrm{cm}^{2}$ and pulse length 27 fs and again we apply the broad window function in the Fourier transform and use perturbation theory for the lowest intensities. The macroscopic conditions are as in Fig. 10; in Fig. 12(a) the position of the gas jet is two mm after the focus and in Fig. 12(b) it is centered in the focus.

As for the longer pulse, the harmonic structure has remarkably emerged after propagation. As discussed above, this is due to suppression of the background radiation by phase matching. When the gas jet is centered in the focus, the individual harmonics, in particular in the plateau, are much broader than for $z_{0}=2$, as we also saw above. This means that radiation from one harmonic actually overlaps with radiation from the next harmonic and these contributions to a certain frequency should therefore be added coherently. However, within the slowly varying envelope approximation approach we are using here, the spectrum of each harmonic is calculated separately and we may have reached the limit of validity of the adiabatic approximation in this case.

\section{CONCLUSIONS}

We have presented a study of high harmonic generation by very short driving pulses. We saw that the harmonic structure, which was nearly absent in the single-atom spectra, emerged after propagation due to the effect of phase match-

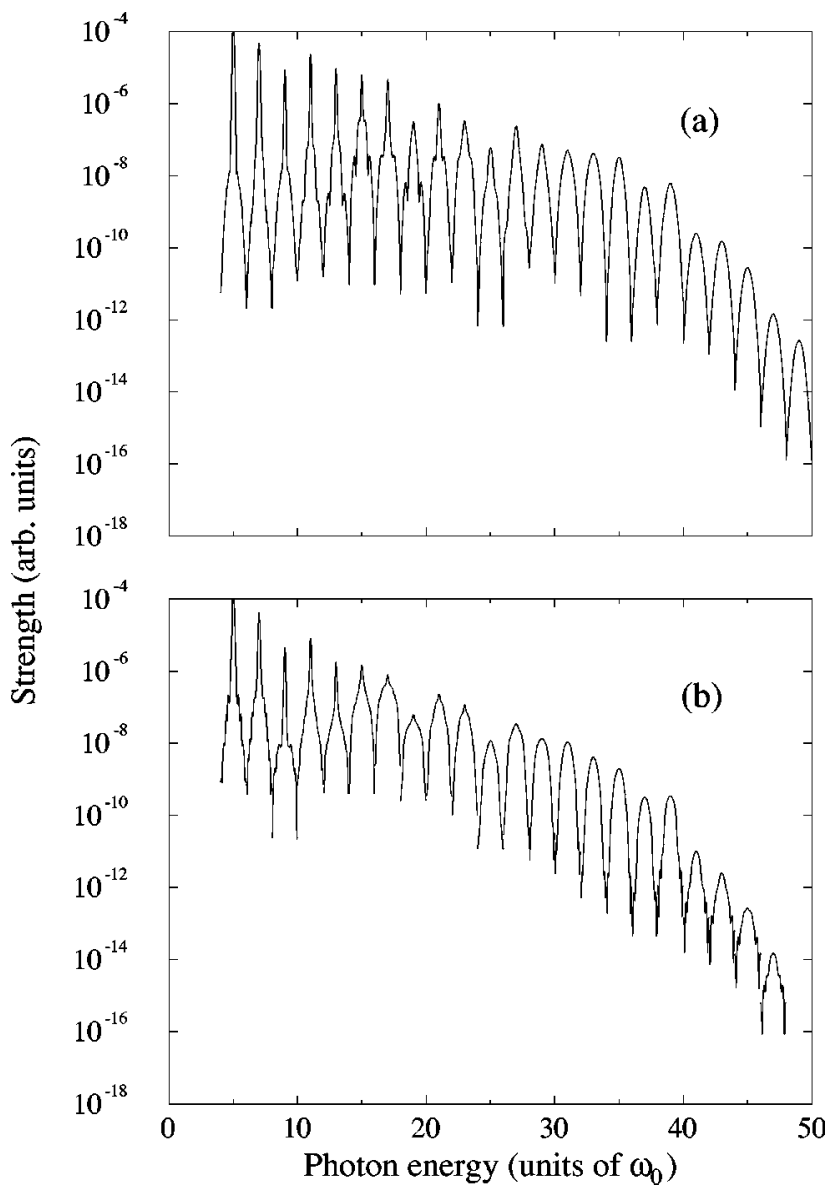

FIG. 12. Spectrum after propagation for $\tau=27 \mathrm{fs}$. In (a) the intensity is $3.5 \times 10^{14} \mathrm{~W} / \mathrm{cm}^{2}$ and $z_{0}=2$ and in (b) the intensity is $3 \times 10^{14} \mathrm{~W} / \mathrm{cm}^{2}$ and $z_{0}=0$.

ing. We saw how the selection by phase matching of one phase behavior compared to another can "clean up" the spectra.

We presented a method to obtain the dipole strength as function of the intensity, from spectra already calculated with the TDSE, for a short driving pulse. This method is based on the adiabatic approximation. We demonstrated that this method works well, within the adiabatic limit of course. This result is satisfying for future computations since it means that for not-too-short pulses one only has to perform the single-atom calculation at one intensity since all the information is contained in one spectrum.

\section{ACKNOWLEDGMENTS}

We wish to thank Philippe Balcou for very enlightening discussions on the dipole phase behavior. Also, discussions with Demetris Lappas and François Salin have been stimulating. This work was carried out in part under the auspices of the U.S. Department of Energy at the Lawrence Livermore National Laboratory under Contract No. W-7405ENG-48. The support of the Swedish Science Research Council, as well as the hospitality of the Institute for Laser Science and Applications at the Lawrence Livermore National Laboratory, is gratefully acknowledged. $\mathrm{Ph}$. Antoine is a collaborateur scientifique du Fonds National de la Recherche Scientifique de la Communauté Française et Belgique. 
[1] J. Zhou, J. Peatross, M. M. Murnane, H. C. Kapteyn, and I. P. Christov, Phys. Rev. Lett. 76, 752 (1996); Z. Chang, A. Rundquist, H. Wang, M. M. Murnane, and H. C. Kapteyn, ibid. 79, 2967 (1997).

[2] Y. Kobayashi, T. Sekikawa, Y. Nabekawa, and S. Watanabe, Opt. Lett. 23, 64 (1998).

[3] Ch. Spielmann, N. H. Burnett, S. Sartania, R. Koppitsch, M. Schnürer, C. Kan, M. Lenzner, P. Wobrauschek, and F. Krausz, Science 278, 661 (1997).

[4] K. J. Schafer and K. C. Kulander, Phys. Rev. Lett. 78, 638 (1997).

[5] I. P. Christov, M. M. Murnane, and H. C. Kapteyn, Phys. Rev. Lett. 78, 1251 (1997).

[6] J. B. Watson, A. Sanpera, K. Burnett, and P. L. Knight, Phys. Rev. A 55, 1224 (1997).

[7] P. B. Corkum, Phys. Rev. Lett. 71, 1994 (1993).

[8] K. J. Schafer, B. Yang, L. F. DiMauro, and K. C. Kulander, Phys. Rev. Lett. 70, 1599 (1993).

[9] W. Becker, S. Long, and J. K. McIver, Phys. Rev. A 50, 1540 (1995), and references therein.

[10] M. Lewenstein, Ph. Balcou, M. Yu. Ivanov, Anne L'Huillier, and P. Corkum, Phys. Rev. A 49, 2117 (1994).

[11] M. Lewenstein, P. Salières, and A. L'Huillier, Phys. Rev. A 52, 4747 (1995).
[12] Ph. Antoine, A. L'Huillier, and M. Lewenstein, Phys. Rev. Lett. 77, 1234 (1996).

[13] Ph. Antoine, D. B. Milosević, A. L’Huillier, M. B. Gaarde, P. Salières, and M. Lewenstein, Phys. Rev. A 56, 4960 (1997).

[14] K. C. Kulander, K. J. Schafer, and J. L. Krause, in Atoms in Intense Radiation Fields, edited by M. Gavrila (Academic, New York, 1992).

[15] P. Salières, A. L'Huillier, P. Antoine, and M. Lewenstein, Adv. At. Mol., Opt. Phys. (to be published).

[16] Ph. Antoine, A. L'Huillier, M. Lewenstein, P. Salières, and B. Carré, Phys. Rev. A 53, 1725 (1995).

[17] C. Kan, C. Capjack, R. Rankin, and N. H. Burnett, Phys. Rev. A 52, R4336 (1995).

[18] Previous studies comparing directly results of the SFA with results of the TDSE approach have shown that the SFA in general reproduces quite accurately the form of the harmonic spectrum for noble-gas atoms, but underestimates the number of photons emitted, typically by a factor of 5-10 [see $\mathrm{Ph}$. Antoine, B. Piraux, D. B. Milosević, and M. Gajda, Laser Phys. 7, 594 (1997)].

[19] Similar results have been obtained using the SFA by P. Salières et al. (unpublished).

[20] C.-G. Wahlström, J. Larsson, A. Persson, T. Starczewski, S. Svanberg, P. Salières, Ph. Balcou, and A. L'Huillier, Phys. Rev. A 48, 4709 (1993). 